\title{
Novel transduction of nutrient stress to Notch pathway by RasGRP3 promotes malignant aggressiveness in human esophageal squamous cell carcinoma
}

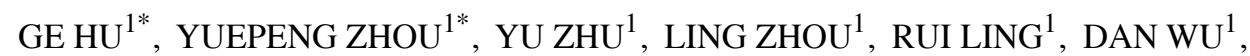 \\ LEI MI ${ }^{1}$, XUEFENG WANG ${ }^{2}$, DONGFANG DAI ${ }^{1}$, CHAOMING MAO $^{1,3}$ and DEYU CHEN ${ }^{1}$ \\ ${ }^{1}$ Institute of Oncology, ${ }^{2}$ Central Laboratory, and ${ }^{3}$ Department of Nuclear Medicine, \\ The Affiliated Hospital of Jiangsu University, Zhenjiang, Jiangsu 212001, P.R. China
}

Received April 6, 2017; Accepted August 28, 2017

DOI: 10.3892/or.2017.5996

\begin{abstract}
In the process of enlarging of tumors, the dissolving tissue structures and remodeling endothelial cells for restoring gas exchange and nutritional support, further facilitate tumor cell invasion and metastasis. Activation of Ras plays a critical role in the development of esophageal squamous cell carcinoma (ESCC), but the underlying mechanisms remain poorly understood. We therefore investigated whether Ras guanyl-releasing protein 3 (RasGRP3), a Ras activator, could promote metastasis by inducing vascular regeneration and further epithelial-mesenchymal transition under nutrient stress (NS). In the present study, we explored that the accumulation of RasGRP3 regulated vascular endothelial growth factor-A production, co-stimulated Notch pathway with high expression of Notch intracellular domain (NICD) and Hes1. Moreover, ESCC cells under NS increased the expression of vimentin, Snail, Slug and MMP9 proteins; while inhibition of Notch activation by DAPT (a $\gamma$-secretase inhibitor) or RasGRP3-targeted RNA interference prevented from the effect. In conclusion, these findings provide a new insight into the upregulation of RasGRP3 involved in Notch pathway activation in the development of ESCC, especially under nutrient deprivation.
\end{abstract}

\section{Instruction}

The estimated number of diagnosed esophageal carcinoma is more than 450,000 per year worldwide, and the largest number

Correspondence to: Dr Deyu Chen or Dr Chaoming Mao, Institute of Oncology, The Affiliated Hospital of Jiangsu University, Jiefang Road 438, Zhenjiang, Jiangsu 212001, P.R. China

E-mail: cdeyu@hotmail.com

E-mail: jq1001@ujs.edu.cn

*Contributed equally

Key words: RasGRP3, nutrient stress, angiogenesis, metastasis, Notch intracellular domain, esophageal squamous carcinoma of cases are esophageal squamous cell carcinoma (ESCC) $(1,2)$. At present, it is considered that ESCC aggresses and spreads by a variety of pathways, including direct invasion, lymphatic and hematogenous metastasis, and the prognosis for metastatic or recurrent ESCC are mostly poor. Due to the high incidence and mortality rates, large susceptible population and limited responses to current therapy causes 5-year survival rate to remain $5-10 \%$ in China (3-5). Although the pathogenesis of ESCC is unclear, it is crucial for the identification of specific biomarkers for accurate evaluation of aggressiveness, elucidating the relevant molecular mechanisms and early detection of tumor recurrence.

Tumors require taking adequate signal factors for unlimited proliferation and distant metastasis, like vascular endothelial growth factor (VEGF), matrix metallopeptidases (MMPs), and directed signaling pathway activation, that all lead to malignance via neovascularization, matrix rearrangement and tumor cell transformation $(6,7)$. In fact, due to the rapid expansion of cancerous cells and relatively hysteresis of blood density in the solid tumor mass, the hypoxia and nutrient deficiency has been identified as crucial incentives (8-12).The altering of nutrients supply can be captured inevitably, but whether it as an independent mechanism affects tumor progression should be studied further. Previous reports showed that Ras protein family members are involved in cellular signal transduction, regulating diverse cell behavior $(13,14)$. In general, once Ras respond to a series of exterior and interior signals, it will subsequently amplify or turn on other factors, which ultimately regulate cell differentiation, growth, invasion, angiogenesis and survival; however, the Ras mutations are rare in tumors (15-17). Moreover, it was suggested that Ras could be constitutively overactivated by various upstream signaling elements, such as Ras guanyl nucleotide releasing peptides (RasGRPs) (18-20). Among them, RasGRP3 promotes guanine nucleotide exchange of H-Ras, R-Ras and Rap1 exerting oncogenic effects; and its overexpression or active form phospho-RasGRP3 were observed in numerous cancer types, regulating the proliferation, survival and migration of human solid tumors and several hematologic neoplasms $(14,21-29)$. In the present study, we investigated whether RasGRP3 also acts as a responder within 
the tumor microenvironment especially under nutrient stress (NS) in human ESCC.

Of note, the presence and activity of Ras induce biological responses closely related with other signaling in cancer progression. For instance, Ras activation leading to upregulation of Notch1 and Notch-mediated oncogenesis requires Ras pathway signaling. Notch signaling is an evolutionary conserved pathway and decides the fate of various cells including cancer cells for a lifetime (30). The interactions between Notch receptors and matched ligands result in the release of Notch intracellular domain (NICD) that translocates to the nucleus and functions as a transcription factor (31). Aberrant Notch signaling has been associated with various cancers; moreover, the high-level expression of Notch1 suggests a poor prognosis for ESCC (32). In some cancer cells, the activation of Notch signaling upregulates and stabilizes Snail under hypoxic conditions or directly stimulates Slug through its promoter while NICD indirectly represses the expression of E-cadherin by binding to its promoter, which form an epithelial-mesenchymal transition (EMT) phenotype (33-35). It is known that Notch pathway is also involved in various steps of vascular development in normal tissue and tumor angiogenesis; and tumor vascularity is associated with aggressive phenotypes including EMT (36,37). Recent evidence indicates that tumor cells with abnormal expression of Notch signaling triggers angiogenesis, and the targeting interruption, such as $\gamma$-secretase inhibitors, may provide effective measures (38). Taken together, Notch signaling as an initiator not only causes malignant aggressiveness but also co-activates with other pathways such as Ras, which might be critical for identifying potential therapeutic targets or modalities.

In the present study, we characterized the expression and functions of RasGRP3 in ESCC specimens and cells, examined the role of RasGRP3 in the activation of angiogenesis and EMT, and studied the Notch signaling pathway that mediated its effects. We found that RasGRP3 was highly expressed in ESCC tumors, involved in the cell migration, invasion of ESCC, regulation of NICD activity and recruiting endothelial cells. In addition, we identified the nutrient stress as a crucial condition, inducing the expression of RasGRP3.

\section{Materials and methods}

Reagents. Penicillin-streptomycin, bull serum albumin (BSA), crystal violet were purchased from Beyotime Institute of Biotechnology (Haimen, China); trypsin was obtained from HyClone Laboratories (Logan, UT, USA). Dulbecco's modified Eagle's medium (DMEM), fetal bovine serum (FBS) were from Gibco (Grand Island, NY, USA). Matrigel was from Invitrogen (Carlsbad, CA, USA). Protease inhibitors were from Merck Millipore (Darmstadt, Germany) and the inhibitor for $\gamma$-secretase, DAPT was obtained from Shanghai Selleck Chemicals Co., Ltd. (Shanghai, China).

Patients and specimens. The Ethics Committees at Affiliated Hospital of Jiangsu University approved this study. From the specimen repository of Pathology Department at Affiliated Hospital of Jiangsu University, 70 patients (48 males and 22 females, from January 2009 to December 2010) and 46 specimen (for co-localization assay, 33 males and 13 females, from
Table I. Specimens assayed for RasGRP3 expression.

\begin{tabular}{|c|c|c|c|}
\hline $\begin{array}{l}\text { Clinicopathological } \\
\text { features }\end{array}$ & $\begin{array}{c}\mathrm{N} \\
(\mathrm{n}=70)\end{array}$ & $\begin{array}{c}\text { RasGRP3 } \\
(\text { mean } \pm \text { SD) }\end{array}$ & P-value \\
\hline Sex & & & 0.578 \\
\hline Male & 48 & $2.87 \pm 1.05$ & \\
\hline Female & 22 & $2.63 \pm 1.21$ & \\
\hline Age (years) & & & 0.625 \\
\hline$\leq 60$ & 9 & $3.01 \pm 1.34$ & \\
\hline$\leq 65$ & 36 & $2.97 \pm 1.46$ & \\
\hline$>65$ & 25 & $3.24 \pm 1.19$ & \\
\hline Tumor location & & & 0.319 \\
\hline Upper/middle & 37 & $4.86 \pm 2.31$ & \\
\hline Lower & 33 & $3.37 \pm 1.18$ & \\
\hline Differentiation & & & 0.3426 \\
\hline Poor & 27 & $3.59 \pm 0.89$ & \\
\hline Moderately & 19 & $2.11 \pm 0.57$ & \\
\hline High & 24 & $3.46 \pm 0.62$ & \\
\hline TNM stage & & & 0.251 \\
\hline I-II & 41 & $4.62 \pm 1.03$ & \\
\hline III-IV & 29 & $4.07 \pm 1.59$ & \\
\hline Lymphatic metastasis & & & 0.012 \\
\hline No & 26 & $1.28 \pm 0.90$ & \\
\hline Yes & 44 & $5.14 \pm 1.56$ & \\
\hline Distant metastasis & & & 0.0239 \\
\hline No & 49 & $1.87 \pm 2.23$ & \\
\hline Yes & 21 & $4.96 \pm 1.27$ & \\
\hline Recurrence & & & 0.037 \\
\hline No & 40 & $3.22 \pm 0.93$ & \\
\hline Yes & 30 & $3.91 \pm 1.66$ & \\
\hline
\end{tabular}

October 2015 to August 2016) with ESCC during surgical resection were identified, written and informed consents were provided by all participants. The main criteria for prognostic evaluation were pathological characteristics (e.g., tumor site, stage, histologic type and size), clinical symptoms (e.g., degree of infiltration and lymph node metastasis) and the 5-year survival rate. The above specific standards were diagnosed as previously described (39). The characteristics of subjects involved in this study are summarized in Table I.

Histological analysis. Immunohistochemical staining was performed on sections fixed and embedded in paraffin as previously described (39). In brief, primary antibodies were rabbit anti-RasGRP3 (Cell Signaling Technology, Beverly, MA, USA, and 1:20 dilution), rabbit anti-phospho-RasGRP3 (Thr133) (Abcam, Cambridge, UK, 1:100 dilution), goat anti-vimentin (Santa Cruz Biotechnology, Santa Cruz CA, USA, 1:50 dilution), rabbit anti-MMP9 and anti-NICD (Cell Signaling Technology, and 1:50 dilution), rabbit anti-VEGFR and anti-Ki-67 (Santa Cruz Biotechnology, 1:50 dilution). Secondary antibodies were HRP anti-mouse, anti-rabbit or anti-goat from R\&D Systems Inc. (Minneapolis, MN, USA) 
at 1:200. All antibodies were diluted in 0.5\% BSA/PBS. HRP-antibodies were visualized using the DAB Substrate kit from Abcam. All slides were under low magnification (x100) to identify, and 4 different regions with the highest vascularity were scanned and blindly analyzed by two investigators using Olympus microscopes connected with a computer running Image-Pro Plus 6.0 software (Media Cybernetics, Rockville, MD, USA). The evaluation criteria for RasGRP3 or other markers were based on our published study: the area was entirely dark brown or $>75 \%$ brown score 3 ; the matrix $>50 \%$ brown was 2 and $>20 \%$ of brown or light brown as 1 while other cases were not scored $(37,39)$. In this study, the average score $>10$ as the strongly positive samples of RasGRP3, $<10$ but $>5$ as moderate, $<5$ but $>2$ as weak samples, while $<2$ as negative.

Microvessel density evaluation by immunofluorescence co-localization. Tissue freshly prepared was submitted from the confirmed ESCC for microvessel density (MVD) immunostaining. Immunohistochemistry and immunofluorescence were applied in frozen sections to evaluate expression and location for RasGRP3 and CD31+ vascular endothelial cells. The primary antibody used was anti-human RasGRP3. The slides were then incubated in HRP anti-mouse followed by DAB Substrate kit. For each sample, after antigen unmasking, the sections were blocked with $2 \%$ BSA in phosphatebuffered saline (PBS) for $1 \mathrm{~h}$ at room temperature. Sections were incubated overnight at $4^{\circ} \mathrm{C}$ with a secondary antibody against CD31 (Santa Cruz Biotechnology, 1:50 in blocking, goat anti-human), followed by incubation for $2 \mathrm{~h}$ with the fluorescence labeling antibody FITC anti-goat (1:200; R\&D Systems). After washed by PBS, sections were viewed under a fluorescence microscope (Olympus, Tokyo, Japan) and counted under x 200 magnification. Any groups of cells that stained positive for CD31 with or without a vessel lumen and distinct from adjacent structures of specimens were counted as a single countable micro-vessel. The score of $\leq 10$ vessels/x 200 magnification was $0 ;>10$ and $\leq 25$ vessels was considered as 1 ; while, the score of $>25$ vessels as 2 . The average of the values obtained by the two reviewers was reported as a single value. The numbers of microvessels in the 4 regions were averaged to give mean MVD score per tumor specimen.

Cell culture and nutrient stress treatment. Human esophageal squamous cancer cells TE-1, TE-10, ECA 109, KYSE-150 and esophageal epithelial cells, Het-1a (Guangzhou Jennio Biotech Co., Ltd., Guangzhou, China), were grown and maintained in DMEM supplemented 10\% FBS. Human umbilical vein endothelial cells (HUVEC-2) were purchased from Guangzhou Jennio Biotech and were maintained in endothelial cell growth Basal Medium-2 supplemented with 10\% FBS. Simultaneously, cancer cells were incubated in the growth medium only containing $1 \%$ FBS for more than 1 month as the nutrient stress treatment. All cells were in a humidified incubator containing $5 \% \mathrm{CO}_{2}$ at $37^{\circ} \mathrm{C}$.

RNA interference. Oligonucleotides for human RasGRP3 siRNA kit were purchased from Shanghai GenePharma Co., Ltd. (Shanghai, China). The kit contains predesigned two siRNAs targeting RasGRP3 gene to ensure work efficiency
(siRNA sense: GCU UAC UUC CUG AGA GCU ATT and antisense: UAG CUC UCA GGA AGU AAG CTT; sense: GGU ACU GGA UUC UGA AGU UTT and antisense: AAC UUC AGA AUC CAG UAC CTT). Cells were transfected with RasGRP3 siRNA or non-specific siRNA ( $2 \mu \mathrm{g} /$ well for 6-well culture plates) using the Opti-MEM (Roche, Mannheim, Germany) plus Lipofectamine 2000 (Thermo Fisher Scientific Inc., Waltham, MA, USA) according to the instruction manual. After 24-h post-transfection, the cells underwent different treatment combinations for ELISA, western blot analysis and cell proliferation assay.

Real-time quantitative PCR analyses. RNA was extracted from ESCC and reverse-transcribed into cDNA as described before. Real-Time PCR was performed using a Biosystems (Bio-Rad Laboratories, Inc., Hercules, CA, USA). Relative expression was calculated by the $2^{-\Delta \Delta C t}$ method using GAPDH as an internal control. The sequences of PCR primers (Invitrogen, Shanghai, China) were as follows: RasGRP3 (forward, 5'-CACGGTCATCAACAAGCACA-3' and reverse 5'-CAGTGTTCGCAGAAGGTTGG-3'); VEGF-A (forward, 5'-AGGAGGAGGGCAGAATCATCA-3' and reverse, 5'-CTC ATTGGATGGCAGTAGCT-3'); Hes1 (forward, 5'-GGTA TTTCCCCAACACGCT-3' and reverse, 5'-GGCAGACATTC TGGAAATGA-3'); Snaill (forward, 5'-CTTCTCCTCTACTT CAGTCTCTTCC-3' and reverse, 5'-TGAGGTATTCCTTGTT GCAGTATTT-3'); Slug (forward, 5'-AACAGAGCATTT GCAGACAGGTC-3' and reverse, 5'-GCTACACAGCAGC CAGATTCC-3'); GAPDH (forward, 5'-TCAACGGATTTG GTCGTATTG-3' and reverse, 5'-TGGGTGGAATCATATTG GAAC-3').

Western blot analysis. Cells were harvested and lysed in a RIPA buffer (CWbiotech, Beijing, China) containing protease inhibitors. Total protein was denatured and separated with 6-12\% SDS-PAGE and further transferred to polyvinylidene difluoride (PVDF) membrane (Thermo Fisher Scientific). After blocking the membrane in 5\% BSA dissolved in TBST, membranes were incubated overnight at $4^{\circ} \mathrm{C}$ with primary antibodies. Afterwards, membranes were incubated with an HRP-conjugated secondary antibody (Cell Signaling Technology) diluted 1:1,000. Target proteins were detected by Pierce ECL Plus Substrate (Thermo Fisher Scientific) and scanned with FluorChem FC3 camera system (Protein-Simple, San Jose, CA, USA).

ELISA. The ELISA analysis of this study was focused on the most important pro-angiogenic factor VEGF-A. The TE-1 cells after nutrient stress treatment, or not, were seeded on 96-well plates (Corning, Inc., Corning, NY, USA) and cultured in serum-free DMEM overnight, then treated with or without RNA interference for 24,48 and $72 \mathrm{~h}$. Culture medium was assayed for VEGF concentration using the antihuman VEGF Immunoassay kit (R\&D Systems) according to the manufacturer's instructions. Briefly, $200 \mu \mathrm{l}$ of control, sample or standard was added to $50 \mu \mathrm{l}$ assay diluent. After 2-h incubation at room temperature, the samples were washed three times. Then, $200 \mu \mathrm{l}$ VEGF conjugate was added for $2 \mathrm{~h}$ and the samples were washed again. After incubation with $200 \mu \mathrm{l}$ substrate solution for $20 \mathrm{~min}, 50 \mu \mathrm{l}$ stop solution 
was added. The concentration of VEGF was measured by the color intensity of solutions using a microplate reader (BioTek Instruments, Inc., Winooski, VT, USA) at 450 and $570 \mathrm{~nm}$, respectively. VEGF concentrations were obtained by comparing the corresponding readings with those of the standard curve using known concentrations of VEGF. Inhibitory rate of secreted VEGF $(\%)=([\mathrm{VEGF}$ content in non-transfected group - VEGF content in transfected group]/ VEGF content in non-transfected group) x 100 .

Cell viability assay. Cell viability was detected by the Cell Counting kit 8 (CCK-8) assay according to the manufacturer's protocol (Biosharp, Hefei, China). Briefly, cells were seeded in 96 -well plates at a density of $4 \times 10^{3}$ cells $/ \mathrm{ml}$, and allowed to adhere for $24 \mathrm{~h}$. Measurement was carried out and cells were treated with the indicated time intervals of si-RasGRP3, then incubated with CCK-8 solution for $3 \mathrm{~h}$ at $37^{\circ} \mathrm{C}$. The optical density (OD) value was measured at an absorbance wavelength of $450 \mathrm{~nm}$ in a microplate reader. All experiments were performed in triplicate.

In vitro tube formation assay. A total of $30 \mu \mathrm{l}$ of Matrigel was added to every well in 96-well plate and incubated at $37^{\circ} \mathrm{C}$ for $30 \mathrm{~min}$ to solidify, and $3 \times 10^{4} \mathrm{HUVECs} /$ well were seeded in medium from TE- 1 cells. The media were as follows: after treatment of nutrient stress, TE-1 cells were seeded on 24-well plates $\left(5 \times 10^{4}\right.$ cells/well) for $24 \mathrm{~h}$. Then, only replaced with a fresh medium or siRNA for human RasGRP3 was transfected according to the above-described method. The next day, the cultured media of different sources were collected. After $12 \mathrm{~h}$ of HUVECs seeding, tube formation was quantified using a microscope (Olympus). Images were analyzed under an inverted microscope at x200 magnification to determine the formation and proportion of branch points and polyhedral closed structures delimiting a lumen. Tube formations were counted from 6 random sites for each well and the experiment was repeated at least three times independently.

Immunofluorescence staining. For immunofluorescence staining, the cells were incubated with primary antibodies against E-cadherin and vimentin (Cell Signaling Technology), followed by incubation with $\mathrm{Cy} 3$ - or FITC-conjugated secondary antibodies (BD Biosciences, San Jose, CA, USA). For upright microscope, the cells grown on coverslips were counterstained with DAPI and were imaged using a fluorescence microscope (Olympus).

Scratch-induced migration assay. TE-1 cells (cultured in $1 \%$ FBS medium more than 1 month) were seeded and synchronized by fresh low serum medium for $8 \mathrm{~h}$. Moreover, the cell monolayers were damaged with a micropipette to create a linear wound $2 \mathrm{~mm}$ in width and were rinsed twice with PBS. The indicated concentrations of siRNA were added and the cells were incubated for $24 \mathrm{~h}$. The scratch phase-contrast images were obtained using a microscope (Olympus), and wound healing was assessed at 5 randomly selected points by measuring the width of the scratch. The results are expressed as the percentage inhibition rate of migration compared with untreated cells using the Adobe Photoshop CS5.
Transwell migration assay. Migration assays were performed as follows: TE-1 and KYSE-150 cells cultured under nutrient stress were transfected with siRNA-RasGRP3 for $24 \mathrm{~h}$. Transwell inserts with a polycarbonate membrane (pore size of $8 \mu \mathrm{m}$; Corning). A density $\sim 1 \times 10^{5}$ of cells was suspended and then seeded in the upper chambers of 24-well plates with FBS-free medium, while medium containing 10\% FBS was deposited in the lower chambers. One-half of the wells with the cells contained the inhibitor DAPT at a final concentration of $10 \mu \mathrm{M}$. After $24 \mathrm{~h}$, cells that migrated were stained by $0.1 \%$ crystal violet and imaged microscopically. Cells were enumerated and the results are expressed as the percentage inhibition rate compared with untreated cells. Each experiment was performed in duplicate.

Statistical analysis. Data are expressed as mean \pm standard deviation (SD). The differences between groups were analyzed using the Student's t-test when only two groups were compared; multiple comparisons were done by either one-way analysis of variance (ANOVA) or ANOVA on ranks. The association between RasGRP3 and the clinicopathological features was assessed using the $\chi^{2}$ test. Recurrence-free survival (RFS) curves were calculated by the Kaplan-Meier methods. $\mathrm{P}<0.05$ was considered statistically significant. These analyses were performed with the SPSS version 16.0.

\section{Results}

RasGRP3 is positively correlated with MVD in human $E S C C$. RasGRP3 has previously shown to be upregulated in human breast, prostate cancer, glioblastoma and melanoma $(22,23,27-29)$, so we also examined the expression of RasGRP3 in ESCC cells using the western blot method. TE-1, TE-10, ECA 109 and KYSE-150 cells detectably express endogenous RasGRP3 and less in Het-1a cells. As mentioned, RasGRP3 is an activator of Ras proteins or pathway. To examine whether RasGRP3 contributes to the activation of Ras pathway in ESCC cells, we set a mimic environment of nutrient deficiency and further examine the potential correlation among RasGRP3, Ras-GTP and total Ras. As shown in Fig. 1A, ESCC cells cultured under the $1 \%$ FBS-containing medium (more than one month) had a significantly increased RasGRP3 and Ras-GTP, however, without any perceptible change in the total Ras. A similar trend of variation was obtained from TE-1 cells stripped of adequate nutrient for 24, $48 \mathrm{~h}$ and 7 days at a relatively short time (data not shown). Since RasGRP3 promotes the proliferation of multiple cancer cells, we also examined the changes of Ki-67 and cell viability after siRNA-RasGRP3 treatment; however, there was no significant change between nutrient stress and normal culture (Fig. 1B and C). In order to avoid the influence caused by apoptosis, the Annexin V-PI assay was also conducted after different treatments, but the differences showed no significance ( $P>0.05$ ) (Fig. 1D). Taken together, the upregulation of RasGRP3 was positively related with nutrient deficiency. Moreover, we found that most of ESCC tumors (reviewing from the paraffin sections of 70 patients) strongly expressed RasGRP3, localized in the cytoplasm, compared to normal adjacent tissues by immunohistochemistry (IHC) staining (Fig. 1E). The intensity of RasGRP3, 
A
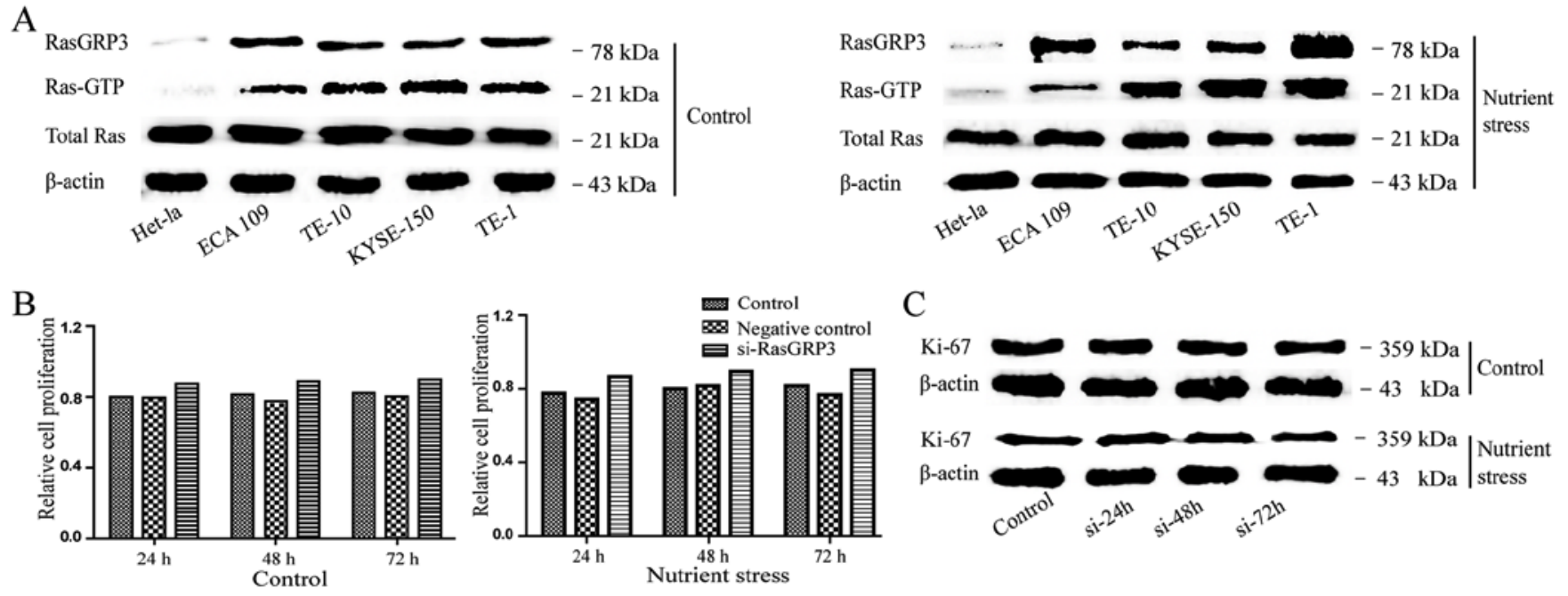

$\mathrm{D}$
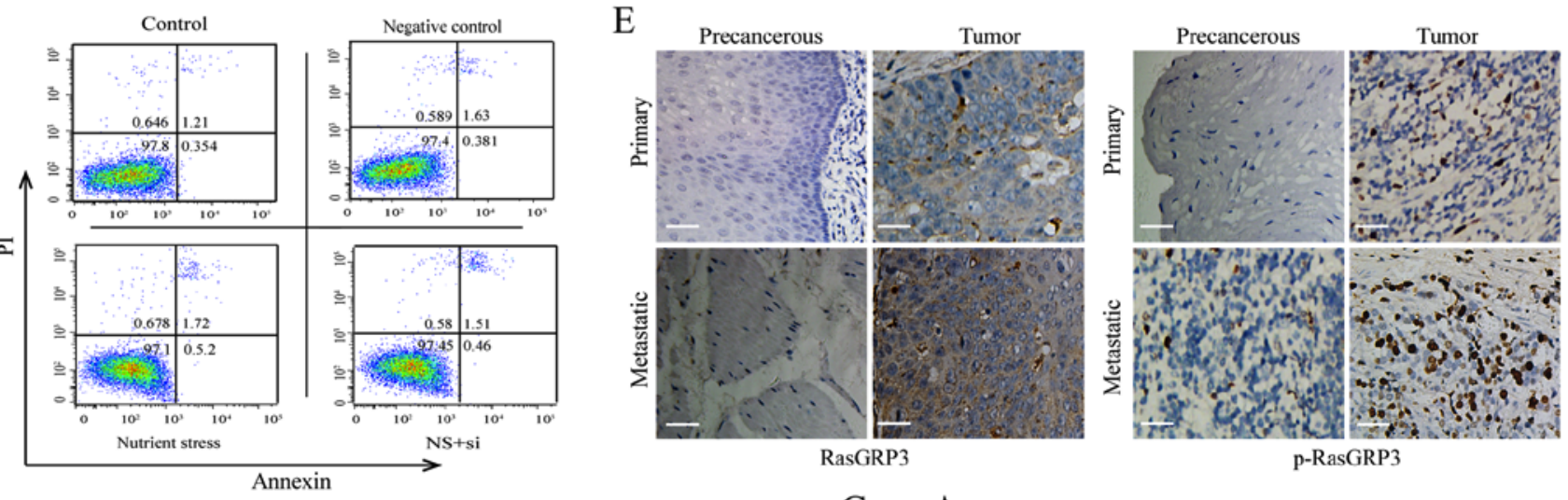

$\mathrm{F}$
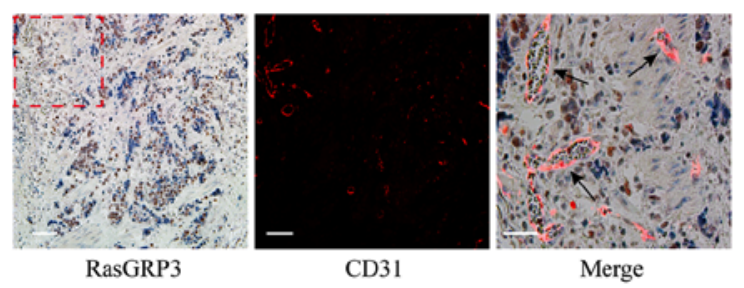

$\mathrm{G}$

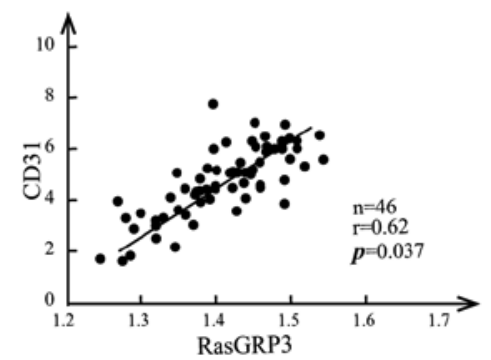

Figure 1. The correlation between RasGRP3 and MVD in esophageal squamous cell carcinoma (ESCC). (A) The expression of RasGRP3 was analyzed by western blot analysis, Ras-GTP and total Ras of ESCC cells lines, TE-1, ECA 109, TE-10 and KYSE-150 and normal esophageal epithelial cells, Het-1a cells under nutrient stress or sufficiency. (B) The viability of TE-1 cells was examined by CCK-8 assay after si-RasGRP3 treatment or not for 24,48 and $72 \mathrm{~h}$ before or after nutrient stress treatment. (C) The expression of Ki-67 in TE-1 cells with or without RasGRP3 interference was measured by immunoblotting under nutrient stress or sufficiency. (D) The percentage of apoptotic cells was detected by flow cytometry that labeled Annexin V-PI. (E) Representative images of RasGRP3 and p-RasGRP3 expression in ESCC tumor and precancerous tissue with or without metastasis (x200; scale bars, $50 \mu \mathrm{m}$ ). (F) The co-location images of IHC (RasGRP3) and IF (Cy3 labelled CD31) in ESCC tumors. Left and middle panel (scale bars, $100 \mu \mathrm{m}$ ). Right panels are magnifications of the area marked by dashed lines (scale bars, $50 \mu \mathrm{m}$ ). (G) The relationship of RasGRP3 and MVD was assessed using Pearson's rank correlation coefficient. All data derive from at least three independent experiments. Significance level, $\mathrm{P}<0.05$.

which was analyzed according to the standard as described in Materials and methods, was independent of patient age, sex, differentiation, tumor location and TNM stage, whereas significantly correlated to the lymphatic or distant metastatic status and recurrence (Table I). In addition, the average nuclear p-RasGRP3 of tumors were higher than precancerous groups, but had no positive correlation with metastasis or recurrence (all $\mathrm{P}>0.05$ ) (data not shown). Because of the deficiency of nutrient has been identified as a trigger to angiogenesis, we also randomly selected and stained 46 frozen specimens of ESCC with antibody CD31 to determine the integrated or anomaly construct of blood vessels by immunofluorescence
(IF). The co-location analysis of IHC and IF revealed a positive correlation between the intensity of RasGRP3 and the number of vessels in human ESCC. As shown in Fig. 1F, which displays a representative sample, we counted the number of vessels per area of RasGRP3 histological sections. There was an average vessel density of 7.6 in RasGRP 3 strongly positive samples, 3.8 in moderate ones and 2.4 for weak positive and 1.8 in negative samples. Throughout the entire comparison process, the intensity of RasGRP3 staining was positively correlative with the increased MVD ( $\mathrm{r}=0.62 ; \mathrm{P}=0.037$; Fig. 1G), suggesting that RasGRP3 plays an important role in the regulation of vascularity in ESCC tissues. 

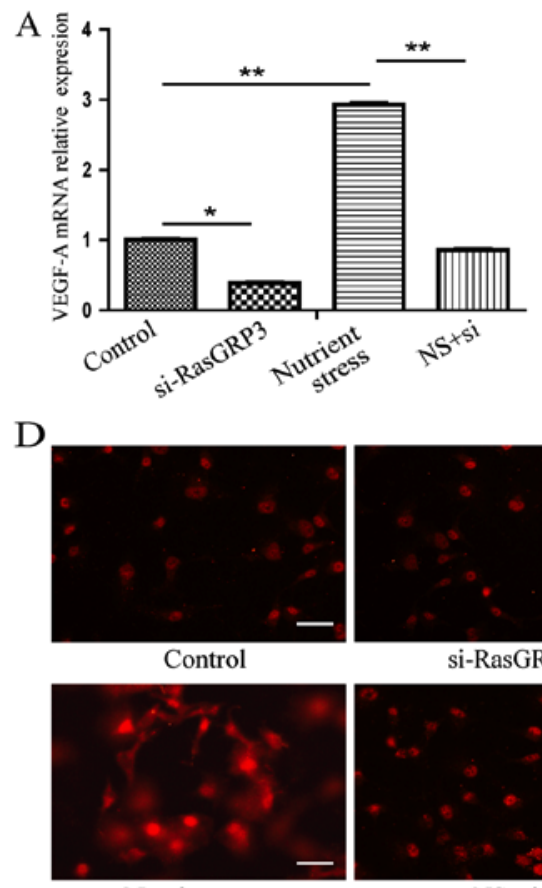

Nutrient stress

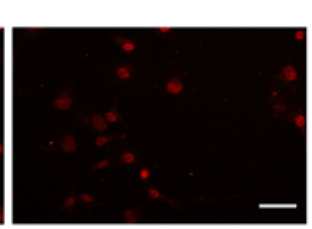

si-RasGRP3

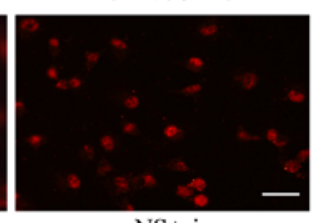

$\mathrm{NS}+\mathrm{si}$
B

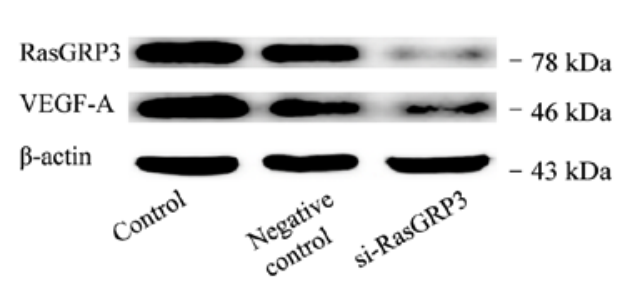

$\mathrm{E}$
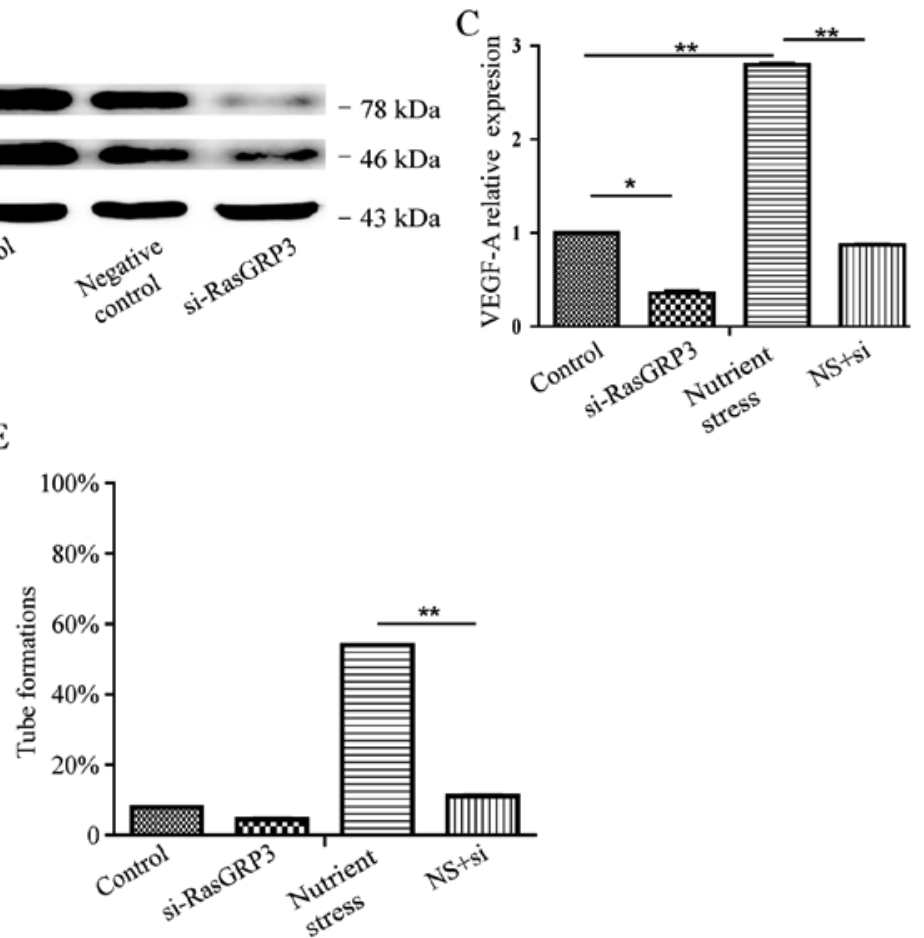

Figure 2. RasGRP3 is involved in angiogenesis stimulated by nutrient stress. (A) TE-1 cells were pretreated of siRNA-RasGRP3 for 24 h or not under nutrient stress or sufficiency, the graph shows the mRNA expression of VEGF-A measured by RT-PCR. The analysis of the CT values was normalized to GAPDH for each sample, and the results are presented as mean \pm SD of three independent experiments. (B) The expressions of RasGRP3 and VEGF-A with siRNARasGRP3 treatment in nutrient deprivation were detected by western blot analysis. (C) The supernatants of TE-1 cells after siRNA-RasGRP3 treatment or not were collected for ELISA analysis (VEGF-A). (D and E) The effect of the supernatant from TE-1 cells treated or untreated by siRNA-RasGRP3 on the ability of HUVECs to form a tube-like network were measured by immunofluorescence (scale bars, $50 \mu \mathrm{m}$ ). Data represent mean values from at least three independent experiments. ${ }^{*} \mathrm{P}<0.05,{ }^{* *} \mathrm{P}<0.01$.

Nutrient stress-instigated RasGRP3 participates in angiogenesis of ESCC. In order to further characterize the contribution of RasGRP3, the factors involved in neovascularization and rearrangement of endothelial cells were quantified. Firstly, the significantly increased VEGF-A mRNA under NS was shown to reduce nearly $70 \%$ after siRNA-RasGRP3 treatment (24 h) (Fig. 2A). Immunoblotting assays showed that the targeted interference at RasGRP3 decreased the expression of VEGF-A protein as compared to TE-1 cells under nutrient deprivation (Fig. 2B). Furthermore, ELISA assay was used to measure the secreted VEGF-A from supernatant of ESCC cells. The result showed that siRNA-RasGRP3 treatment reversed the significant increase of VEGF-A caused by chronic low-serum culturing (Fig. 2C). Given the role that restriction of nutrient might regulate angiogenesis via rearranging endothelial cells with the increased VEGF-A, we sought to determine the effect of RasGRP3 on tube formation of HUVECs. As shown in Fig. 2D, culture supernatant from TE-1 cells under NS induced complete tube formation, whereas cells after pre-knockdown of RasGRP3 suppressed the formation of robust tube at $12 \mathrm{~h}$ (Fig. 2E; P<0.01). Taken together, these data suggested that RasGRP3 as a crucial factor could mediate angiogenic signaling under the nutrient stress.

RasGRP3 triggers the EMT and finally metastasis of ESCC cells under nutrient stress. Although RasGRP3 contributes to the angiogenesis of ESCC, the focus of this study so far has been on its role in further malignant aggressiveness. Therefore, we tested whether there were differences in EMT-related proteins due to the change of RasGRP3. Firstly, the figures based on immunofluorescence staining of E-cadherin and vimentin indicated that there were significance differences between NS treatment or not. However, the RNA interference targeting RasGRP 3 could inhibit the downregulation of E-cadherin and upregulate vimentin caused by NS (Fig. 3A). To go a step further, we also validated the activation of EMT by detecting the expression of Snail1, Slug and Hes1. The expression of Snail 1, Slug and Hes1 mRNA in TE-1 cells under NS were downregulated to $\sim 20 \%$ after siRNA-RasGRP3 treatment (P<0.01; Fig. 3B). The cellular morphology transformation and increased expression of MMPs are related to the acquisition of invasiveness and metastasis in various cancer cells. To investigate the effects of RasGRP3 on human ESCC cells invasion and metastasis, we subsequently analyzed the expression of E-cadherin, vimentin and MMP9 by western blot analysis (Fig. 3C). The decreased levels of vimentin and MMP9 in combination with upregulation of E-cadherin after siRNA-RasGRP3 treatment suggested that RasGRP3 regulated the progress of EMT. In the present study, RasGRP3 accumulation also upregulated the expression of NICD, whereas had no effect on Notch1 protein level (Fig. 3C). Then, we found that suppression of the Notch signaling by DAPT $(10 \mu \mathrm{M})$ or siRNA-RasGRP3 decreased levels of vimentin, MMP9 and NICD induced by NS (Fig. 3C). Moreover, carcinoma cells produce a whole range of proteases that degrade the extracellular matrix and basement membranes, which has been regarded as initial steps in the process of invasion. Therefore, we determined ESCC 
A

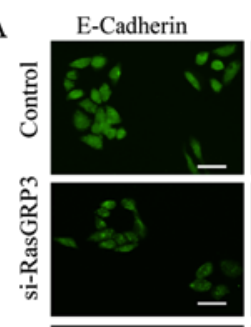

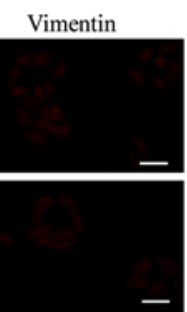
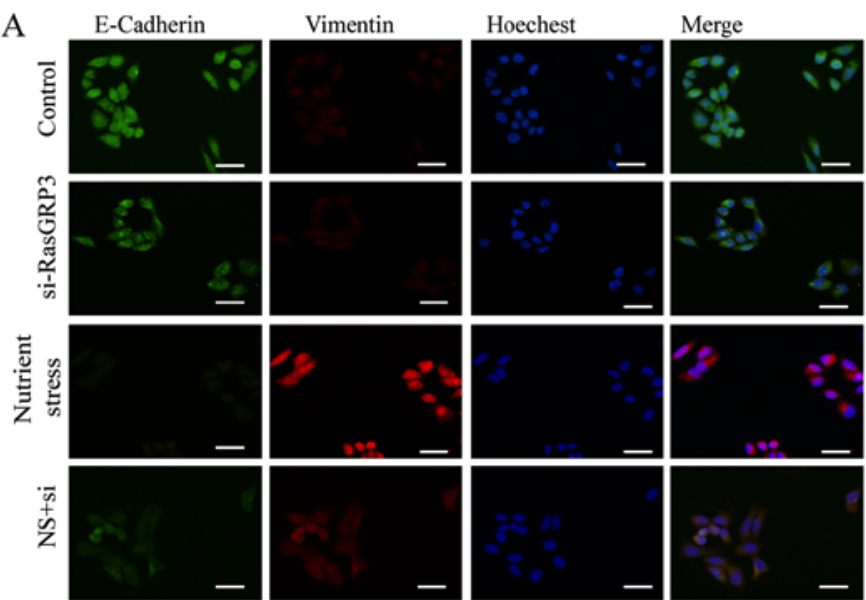

B

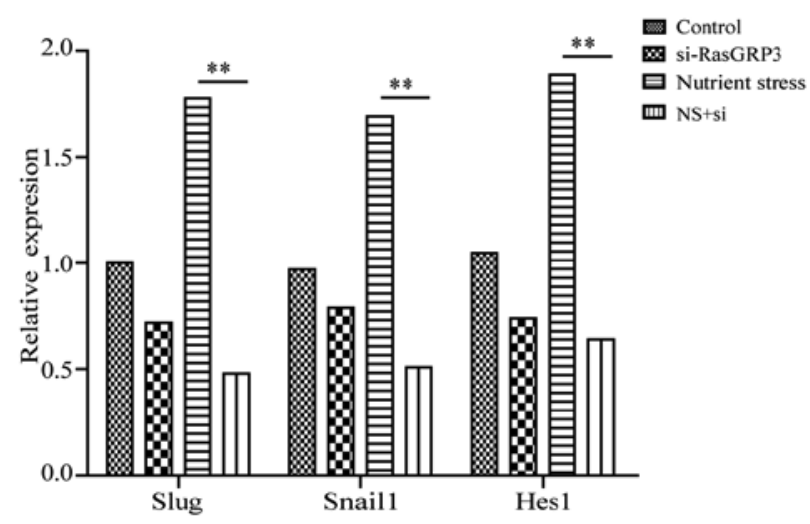

C

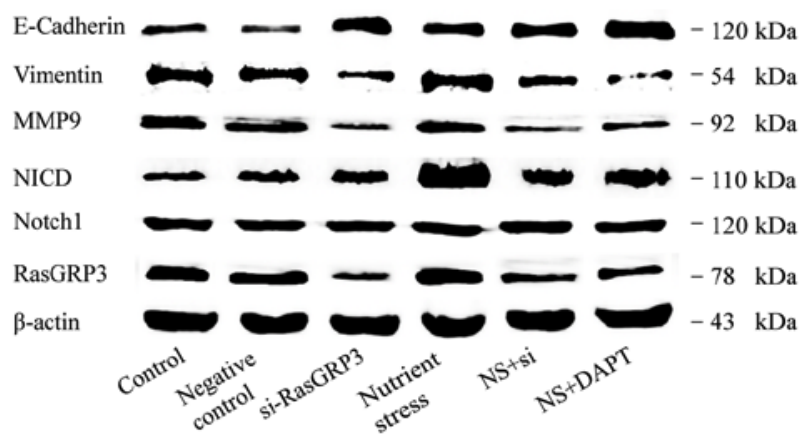

D

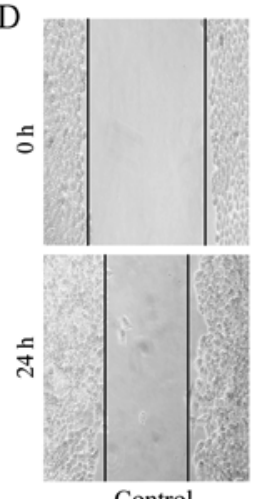

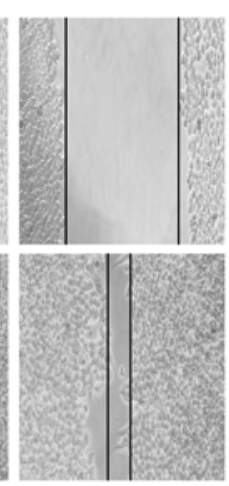

Nutrient stress

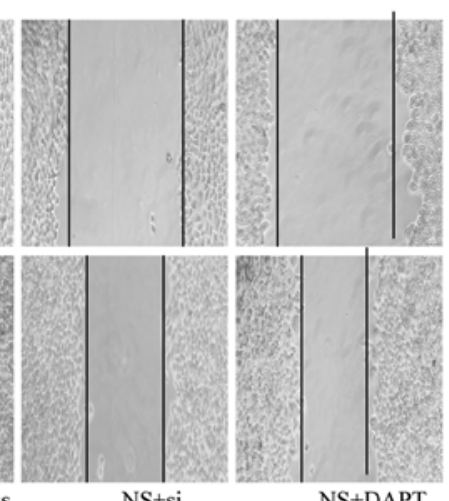

NS+DAPT
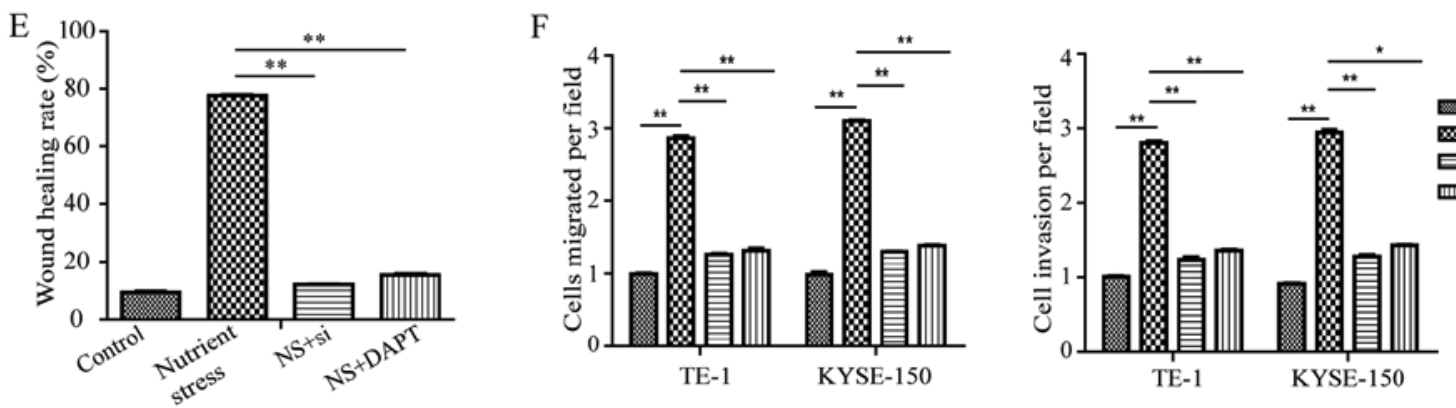

Figure 3. The nutrient stress-derived EMT was depended on the accumulation of RasGRP3. (A) The cells cultured under nutrient stress or sufficiency were treated with either siRNA-RasGRP3 or nonsense siRNA for $24 \mathrm{~h}$, and then stained with E-cadherin and vimentin antibodies for immuno-colocalization. E-cadherin marked as green and vimentin as red (scale bars, $50 \mu \mathrm{m}$ ). (B) Pretreated with siRNA-RasGRP3, the mRNA levels of Snaill, Slug and Hes-1 of TE-1 cells were measured by RT-PCR (mean \pm SD). GAPDH was used as the housekeeping control. (C) E-cadherin, vimentin, MMP-9, NICD, Notch1 and RasGRP3 expression levels in TE-1 cells treated with DAPT (50 ng/ml) for $24 \mathrm{~h}$ or siRNA-RasGRP3 were detected by western blot analysis. (D) Representative wounds of the monolayers of ESCC cells treated with siRNA-RasGRP3 or DAPT $(50 \mathrm{ng} / \mathrm{ml})$ are shown in comparison to untreated controls. (E) The values are expressed as wound healing rate (mean $\pm \mathrm{SD}$ ). (F) Comparisons of migration and invasion abilities after siRNA-RasGRP3 or DAPT (50 ng/ml) compared to untreated controls in TE- 1 and KYSE 150 cells. Quantitative analysis of panels are shown in the bar chart. ${ }^{*} \mathrm{P}<0.05,{ }^{* *} \mathrm{P}<0.01$.

cells by scratch-induced migration assay (Fig. 3D). As shown in Fig. 3E, the TE-1 cells under NS migrated more rapidly than that in control groups, and the final distance of cells from the starting wound edge were increased by $58 \%(\mathrm{P}<0.05)$; however, the number was back to $12 \%$ for cells transfected with interference fragment of RasGRP3. To further investigate the impact of RasGRP3 on migration, Transwell assays were performed. Approximately 3-fold increases of invasion were observed under NS compared with control groups $(\mathrm{P}<0.01)$. In keeping with above results, knockdown of RasGRP3 or DAPT treatment showed a significant decrease of the abilities of migration and invasion (Fig. 3E). Collectively, these results indicated that RasGRP3 promotes metastasis dependent on the activity of Notch pathway.

Association of RasGRP3 and clinicopathological features with prognosis in human ESCC samples. In order to address the diagnostic and prognostic value of RasGRP3, precancerous or tumor serial sections of ESCC were stained with VEGFR, Ki-67, vimentin, MMP9 and NICD antibody to quantify the potential correlation. Among 70 patients with ESCC, 34 samples expressed with a high level of RasGRP3, 32 samples expressed at moderate intensity, whereas 4 samples with lower or negative staining. The expression of vimentin and MMP9 


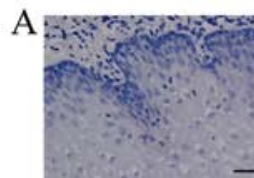

Precancerous

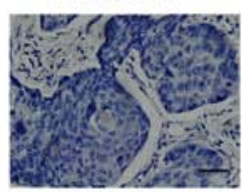

Tumor

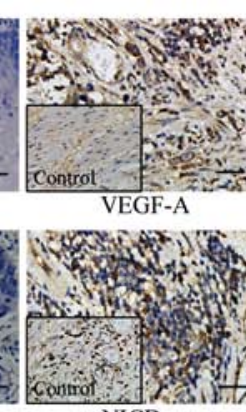

NICD

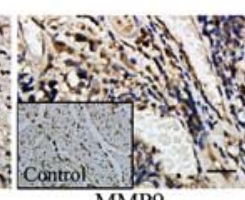
MMP9

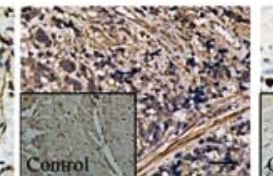

Vimentin

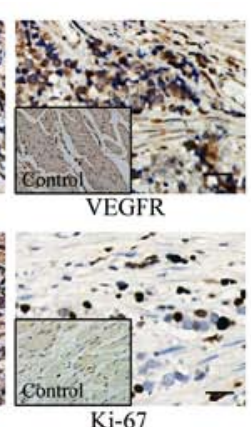

B

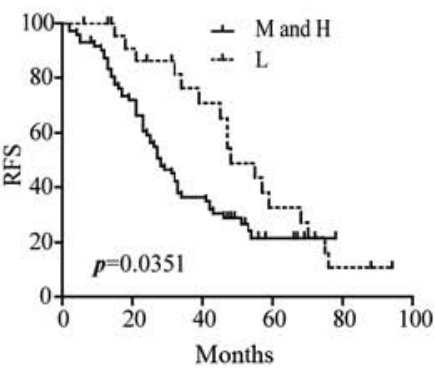

C

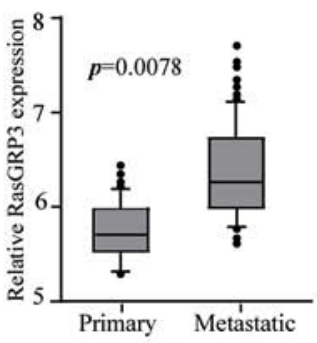

Figure 4. The relationship between clinicopathological features, prognosis and levels of RasGRP3 in human ESCC. (A) The representative IHC results show the expression of VEGF-A, VEGFR, Ki-67, vimentin, MMP9 and NICD in ESCC tissues. Left panels are negative controls, and the inset images are control tissues (x200; scale bars, $50 \mu \mathrm{m}$ ). (B) Kaplan-Meier survival curves of the 5-year RFS for ESCC patients stratified by RasGRP3 expression. M and H, the moderate and high expression of RasGRP3; L, lower expression of RasGRP3. (C) Based on home interview and IHC results of ESCC patients, the metastasis rate and effects of RasGRP3 were analyzed by correlation analysis. Significance level, $\mathrm{P}<0.05$.

Table II. Association between RasGRP3 expression and clinicpathological features with prognosis.

\begin{tabular}{llcl}
\hline & M and $\mathrm{H}$ & L and N & P-value \\
\hline Vimentin & $78 \%$ & $11 \%$ & 0.028 \\
MMP9 & $76.9 \%$ & $14 \%$ & 0.019 \\
NICD & $80 \%$ & $6 \%$ & 0.000 \\
Ki-67 & $54 \%$ & $50 \%$ & 0.506 \\
VEGFR & $64 \%$ & $67 \%$ & 0.319 \\
\hline
\end{tabular}

$\mathrm{M}$ and $\mathrm{H}$, moderate and high RasGRP3 expression; $\mathrm{L}$ and $\mathrm{N}$, lower and negative RasGRP3 expression.

showed moderate or high representation in $78 \%$ and $76.9 \%$ of RasGRP3 stable positive samples (the 34 high and 32 moderate sections), with 11 and $14 \%$ in the remaining 4, respectively. NICD was strongly positive in $\sim 80 \%$ of RasGRP3 stable positive samples, compared with $6 \%$ in lower or negative sections. However, Ki-67 was densely labeled among 54\% of stable RasGRP3 positive samples and 50\% of remaining 4 samples. Furthermore, data showed no significant differences of VEGFR among the samples (64\% positive staining with stable RasGRP3 and 67\% for lower and negative expression) (Fig. 4A and Table II). In Kaplan-Meier survival curves, patients with high and moderate expression of RasGRP3 showed a worse outcome, and the lower expression group had a longer survival time $(\mathrm{P}=0.0351$; Fig. 4B). According to the log-rank method, the metastasis rates were $61.0 \%$ for patients with moderate and high RasGRP3 expression, compared with $29.8 \%$ with lower expression ( $\mathrm{P}=0.0078$; Fig. 4C). Thus, RasGRP3 appears to be a strong marker of poor prognosis in patients of ESCC.

\section{Discussion}

It is evident that enough vessels are the mechanical approach for tumor cell migration across vascular wall, restoration of oxygen and nutritional provision (37). Therefore, an appropriate definition to label blood supply along with cancer progression contributes to diagnostic and guiding therapeutic treatment. Recent research has shown that Ras is one of the important stress-response genes, and its activation is attributed to upstream signaling such as tyrosine kinase receptors, excessive activation of guanine nucleotide exchange factors (GEFs) $(13,14,16,17,22-29)$. As mentioned, RasGRP, as a GEFs in various tissues and tumors, not only have the structures for binding diacylglycerol (DAG) but also modulates Ras pathway activity and even malignant transformation processes. For instance, RasGRPs can function as oncogenes in multiple cancers, and an elevated expression of RasGRP3 have been found in Burkitt's lymphoma, B cell leukemia, breast, prostate cancer, glioblastoma and melanoma, affecting the proliferation and treatment-resistance (22-29). Here, we found that RasGRP3 and p-RasGRP3 were expressed higher in ESCC specimens comparing to the peritumoral normal tissues. Moreover, the treatment of nutrient stress, whether at a short or long time, increased RasGRP3 of ESCC cells, contributing to the activation of Ras-GTP. These results suggest that RasGRP3 have the indicative function for malignant aggressiveness. Note that an existing study have confirmed RasGRP3 can be upregulated by VEGF in angiogenesis and promoted cancer multiplication, invasion and metastasis (40). VEGF, as one of the most potent growth factors, mediating angiogenesis via recruiting and stimulating the growth of endothelial cells, leading to increased vascularity (41). Thus, in order to refine the patient population of ESCC most likely to respond to high RasGRP3, we performed MVD analyses using section from the original esophagectomy blocks, and data based on pathologic analysis showed a close relationship between MVD and RasGRP3. For further confirmation, experiments in vitro have also investigated RNA and protein of VEGF-A which were mediated by the accumulation of RasGRP3 after chronic low-serum treatment. However, another way for tumors to expand is by proliferation, dependent on the wellknown proliferation marker of Ki-67 and cell viability. Our data have shown that pretreatment of ESCCs with siRNARasGRP3 do not suppress proliferation or induce apoptosis either with or without nutrient deprivation. In patients who presented with high levels of RasGRP3, we observed no consistent increases of Ki-67 also, surprisingly, no positive correlation to VEGFR, which has been proved to cause new tubule formation, while the accumulated RasGRP3 enhanced 
tube formation of HUVECs in vitro (41). Therefore, even though RasGRP3 has been shown to take part in the angiogenesis, but as a complex process taking several steps, the disparity and significance are worthy to study further.

The reasons why ESCC caused recurrence, spreading and metastasis remain unknown. We presumed that this could be explained by abnormal microenvironment and signaling dysfunction. It is well-known that cancer cells more easily undergo EMT during penetration through vascular wall, causing an increase of circulating cells and metastasis (42). At the cellular level, RasGRP3 showed no effect on proliferation but amplified the process of mesenchymal transition, downregulated the E-cadherin as well as increased the expression of Snail1, Slug, vimentin and MMP9 in TE-1 cells. Moreover, these effects were consistent with ESCC tumor samples, showing that the strong expression and co-localization of RasGRP3 with vimentin and MMP9. Considering the risks and impacts of EMT in ESCC, we performed a regression analysis and observed an earlier relapse to lymph nodes and organs in patients who presented with high levels of RasGRP3. A median survival of 32 months in patients displaying steady RasGRP3 (strongly and moderate positive) compared to patients more than five years who exhibited low or no RasGRP3. Indeed, for the activation of RasGRP3 is likely a dynamic process, the expression of p-RasGRP3 was not representative among ESCC with different degrees, however, the abnormal RasGRPs activation contributes to oncogenesis via affecting the regulation of multiple downstream molecules (21-23,29). To explore the mechanism underlying RasGRP3-induced angiogenesis and metastasis in ESCC, we examined its effects on Notch signaling, known to be activated by the Ras protein family, although Ras mutations are not common in cancers $(15,30)$. Aberrant Notch signaling has been associated with the development and progression of various cancers, high-level expression of Notch1 and its ligand Jagged1 are predictive of poor survival and recurrence in $\operatorname{ESCC}(32,43)$. Consistent with this, we found that the RasGRP3 was mostly localized in the cytoplasm whereas NICD showed nuclear staining in ESCC tumors. As presented, the downregulation of RasGRP3 caused a significant decrease of Hes-1 in TE-1 cells. Of relevance, it was confirmed that modulation of RasGRP3 contributes to Akt and ERK1/2 activation in prostate cancer and melanoma cells, we are the first to demonstrate that RasGRP3 modulated the enrichment of NICD and contributed the activation of Notch under nutrient stress (23). Accordingly, we propose that RasGRP3 promoted angiogenesis, migration and invasion of ESCC cells via upregulation of the Notch pathway. The activation of Ras led to upregulation of NICD, and Notch pathway mediated oncogenesis required Ras signals, suggesting an association between these two pathways.

Thus, the nutrient stress is an independent factor, and has an influence on tumour growth through biological targeting of malignant aggressiveness. In the present study, we sought to investigate the consequences of coordinate activation of Notch and Ras pathways on the survival of ESCC patients, and the influence of RasGRP3 on ESCCs. In conclusion, the $\gamma$-secretase inhibitor, DAPT, was shown to have sufficient activity against aggressiveness in esophageal squamous cell carcinoma, which provides a potential treatment strategy for ESCC patients with metastasis under nutrient stress.

\section{Acknowledgements}

The present study was supported by the National Natural Science Foundation of China (nos. 81572956 and 81370889), and the Wu Jieping Medical Foundation (no. 320.6755.15022).

\section{References}

1. Ferlay J, Shin HR, Bray F, Forman D, Mathers C and Parkin DM: Estimates of worldwide burden of cancer in 2008: GLOBOCAN 2008. Int J Cancer 127: 2893-2917, 2010.

2. Siegel RL, Miller KD and Jemal A: Cancer statistics, 2015. CA Cancer J Clin 65: 5-29, 2015.

3. Chen MF, Yang YH, Lai CH, Chen PC and Chen WC: Outcome of patients with esophageal cancer: A nationwide analysis. Ann Surg Oncol 20: 3023-3030, 2013.

4. Lin Y, Totsuka Y, He Y, Kikuchi S, Qiao Y, Ueda J, Wei W, Inoue $\mathrm{M}$ and Tanaka $\mathrm{H}$ : Epidemiology of esophageal cancer in Japan and China. J Epidemiol 23: 233-242, 2013.

5. Wu C, Hu Z, He Z, Jia W, Wang F, Zhou Y, Liu Z, Zhan Q, Liu Y, Yu D, et al: Genome-wide association study identifies three new susceptibility loci for esophageal squamous-cell carcinoma in Chinese populations. Nat Genet 43: 679-684, 2011.

6. Akakura N, Kobayashi M, Horiuchi I, Suzuki A, Wang J, Chen J, Niizeki H, Kawamura K, Hosokawa M and Asaka M: Constitutive expression of hypoxia-inducible factor-1alpha renders pancreatic cancer cells resistant to apoptosis induced by hypoxia and nutrient deprivation. Cancer Res 61: 6548-6554, 2001.

7. Catalano V, Turdo A, Di Franco S, Dieli F, Todaro M and Stassi G: Tumor and its microenvironment: A synergistic interplay. Semin Cancer Biol 23: 522-532, 2013.

8. Ghosh R, Lipson KL, Sargent KE, Mercurio AM, Hunt JS, Ron D and Urano F: Transcriptional regulation of VEGF-A by the unfolded protein response pathway. PLoS One 5: e9575, 2010.

9. Horsman MR and Vaupel P: Pathophysiological basis for the formation of the tumor microenvironment. Front Oncol 6: 66, 2016.

10. Kranenburg O, Gebbink MF and Voest EE: Stimulation of angiogenesis by Ras proteins. Biochim Biophys Acta 1654: 23-37, 2004.

11. Ojha R, Bhattacharyya S and Singh SK: Autophagy in Cancer stem cells: A potential link between chemoresistance, recurrence, and metastasis. Biores Open Access 4: 97-108, 2015.

12. Xu Y, Xia X and Pan H: Active autophagy in the tumor microenvironment: A novel mechanism for cancer metastasis. Oncol Lett 5: 411-416, 2013.

13. Etienne-Manneville S and Hall A: Rho GTPases in cell biology. Nature 420: 629-635, 2002.

14. Stone JC: Regulation of Ras in lymphocytes: Get a GRP. Biochem Soc Trans 34: 858-861, 2006.

15. Downward J: Targeting RAS signalling pathways in cancer therapy. Nat Rev Cancer 3: 11-22, 2003.

16. Kolch W: Meaningful relationships: The regulation of the Ras/ Raf/MEK/ERK pathway by protein interactions. Biochem J 351: 289-305, 2000.

17. Zuber J, Tchernitsa OI, Hinzmann B, Schmitz AC, Grips M, Hellriegel M, Sers C, Rosenthal A and Schäfer R: A genomewide survey of RAS transformation targets. Nat Genet 24: 144-152, 2000.

18. Garcia LC, Donadío LG, Mann E, Kolusheva S, Kedei N, Lewin NE, Hill CS, Kelsey JS, Yang J, Esch TE, et al: Synthesis, biological, and biophysical studies of DAG-indololactones designed as selective activators of RasGRP. Bioorg Med Chem 22: 3123-3140, 2014.

19. Hennig A, Markwart R, Esparza-Franco MA, Ladds G and Rubio I: Ras activation revisited: Role of GEF and GAP systems. Biol Chem 396: 831-848, 2015.

20. Roberts PJ and Der CJ: Targeting the Raf-MEK-ERK mitogenactivated protein kinase cascade for the treatment of cancer. Oncogene 26: 3291-3310, 2007.

21. Frau M, Feo F and Pascale RM: Pleiotropic effects of methionine adenosyltransferases deregulation as determinants of liver cancer progression and prognosis. J Hepatol 59: 830-841, 2013.

22. Lee HK, Finniss S, Cazacu S, Xiang C, Poisson LM, Blumberg PM and Brodie C: RasGRP3 regulates the migration of glioma cells via interaction with Arp3. Oncotarget 6: 1850-1864, 2015. 
23. Nagy Z, Kovács I, Török M, Tóth D, Vereb G, Buzás K, Juhász I, Blumberg PM, Bíró T and Czifra G: Function of RasGRP3 in the formation and progression of human breast cancer. Mol Cancer 13: 96, 2014.

24. Porpaczy E, Bilban M, Heinze G, Gruber M, Vanura K, Schwarzinger I, Stilgenbauer S, Streubel B, Fonatsch C and Jaeger U: Gene expression signature of chronic lymphocytic leukaemia with Trisomy 12. Eur J Clin Invest 39: 568-575, 2009.

25. Teixeira C, Stang SL, Zheng Y, Beswick NS and Stone JC: Integration of DAG signaling systems mediated by PKC-dependent phosphorylation of RasGRP3. Blood 102: 1414-1420, 2003

26. Yamagishi M, Katano H, Hishima T, Shimoyama T, Ota Y, Nakano K, Ishida T, Okada S and Watanabe T: Coordinated loss of microRNA group causes defenseless signaling in malignant lymphoma. Sci Rep 5: 17868, 2015.

27. Yang D, Kedei N, Li L, Tao J, Velasquez JF, Michalowski AM, Tóth BI, Marincsák R, Varga A, Bíró T, et al: RasGRP3 contributes to formation and maintenance of the prostate cancer phenotype. Cancer Res 70: 7905-7917, 2010.

28. Yang D, Tao J, Li L, Kedei N, Tóth ZE, Czap A, Velasquez JF, Mihova D, Michalowski AM, Yuspa SH, et al: RasGRP3, a Ras activator, contributes to signaling and the tumorigenic phenotype in human melanoma. Oncogene 30: 4590-4600, 2011.

29. Zeng X, Hu Z, Wang Z, Tao J, Lu T, Yang C, Lee B and Ye Z: Upregulation of RASGRP3 expression in prostate cancer correlates with aggressive capabilities and predicts biochemical recurrence after radical prostatectomy. Prostate Cancer Prostatic Dis 17: 119-125, 2014

30. Weijzen S, Rizzo P, Braid M, Vaishnav R, Jonkheer SM, Zlobin A, Osborne BA, Gottipati S, Aster JC, Hahn WC, et al: Activation of Notch-1 signaling maintains the neoplastic phenotype in human Ras-transformed cells. Nat Med 8: 979-986, 2002.

31. Takebe N, Nguyen D and Yang SX: Targeting notch signaling pathway in cancer: Clinical development advances and challenges. Pharmacol Ther 141: 140-149, 2014.

32. Ogawa $\mathrm{R}$, Ishiguro $\mathrm{H}$, Kimura $\mathrm{M}$, Funahashi $\mathrm{H}$, Wakasugi $\mathrm{T}$, Ando T, Shiozaki M and Takeyama H: NOTCH1 expression predicts patient prognosis in esophageal squamous cell cancer. European surgical research. Eur Surg Res 51: 101-107, 2013.
33. Lim SO1, Gu JM, Kim MS, Kim HS, Park YN, Park CK, Cho JW, Park YM and Jung G: Epigenetic changes induced by reactive oxygen species in hepatocellular carcinoma: methylation of the E-cadherin promoter. Gastroenterology 135: 2128-2140, 2140. e2121-2128, 2008.

34. Sahlgren C, Gustafsson MV, Jin S, Poellinger L and Lendahl U: Notch signaling mediates hypoxia-induced tumor cell migration and invasion. Proc Natl Acad Sci USA 105: 6392-6397, 2008

35. Shao S, Zhao X, Zhang X, Luo M, Zuo X, Huang S, Wang Y, $\mathrm{Gu} S$ and Zhao X: Notch1 signaling regulates the epithelialmesenchymal transition and invasion of breast cancer in a Slug-dependent manner. Mol Cancer 14: 28, 2015.

36. Benedito R, Roca C, Sörensen I, Adams S, Gossler A, Fruttiger M and Adams RH: The notch ligands Dll4 and Jagged1 have opposing effects on angiogenesis. Cell 137: 1124-1135, 2009.

37. Noguera-Troise I, Daly C, Papadopoulos NJ, Coetzee S, Boland P, Gale NW, Lin HC, Yancopoulos GD and Thurston G: Blockade of Dl14 inhibits tumour growth by promoting non-productive angiogenesis. Nature 444: 1032-1037, 2006.

38. Dorneburg C, Goß AV, Fischer M, Roels F, Barth TF, Berthold F, Kappler R, Oswald F, Siveke JT, Molenaar JJ, et al: $\gamma$-Secretase inhibitor I inhibits neuroblastoma cells, with NOTCH and the proteasome among its targets. Oncotarget 7: 62799-62813, 2016.

39. Chen D, Li W, Liu S, Su Y, Han G, Xu C, Liu H, Zheng T, Zhou Y and Mao C: Interleukin-23 promotes the epithelial-mesenchymal transition of oesophageal carcinoma cells via the $\mathrm{Wnt} / \beta$-catenin pathway. Sci Rep 5: 8604, 2015.

40. Roberts DM, Anderson AL, Hidaka M, Swetenburg RL, Patterson C, Stanford WL and Bautch VL: A vascular gene trap screen defines RasGRP3 as an angiogenesis-regulated gene required for the endothelial response to phorbol esters. Mol Cell Biol 24: 10515-10528, 2004.

41. Ferrara N, Gerber HP and LeCouter J: The biology of VEGF and its receptors. Nat Med 9: 669-676, 2003.

42. Meng F and Wu G: The rejuvenated scenario of epithelialmesenchymal transition (EMT) and cancer metastasis. Cancer Metastasis Rev 31: 455-467, 2012.

43. Yuan Y, Hu Y, Zhao Y and Chen L: Expressions of Notch signaling-associated proteins in esophageal squamous cell carcinoma. Zhonghua Wei Chang Wai Ke Za Zhi 18: 909-913, 2015 (In Chinese). 